Überlegungen zum Verhältnis von Umwelt und Innovation

\title{
Prometheus läßt grüßen
}

\section{Die Ambivalenz des technischen Fortschritts wird zur Zeit wieder eher unter positiven Vorzeichen gedeutet. Eine Reihe von aktuellen Forschungsprojekten beschäftigt sich sich mit der Hypothese, daß Umweltinnovationen sowohl öko- logisch als auch ökonomisch vorteilhaft sind. Während die weiteren Beiträge des Heftes die verschiedenen Facetten beleuchten, wird im folgenden Überblick eine kritische Bilanz gezogen und weitere Forschungsperspektiven aufgezeigt.}

Von Stefan Zundel

$\mathrm{D}$ as Feuer ist eine der ersten Erfindungen der Menschheit. In der griechischen Sagenwelt ist es Prometheus, der dem Göttervater Zeus das Feuer stiehlt. Er ermöglicht - modern gesprochen - den Menschen, ein bislang unkontrollierbares Naturphänomen $z u$ beherrschen und den eigenen Zwecken dienstbar zu machen. Den Erfolg der Erfindung, der in der Perspektive der griechischen Mythologie ein Schritt der Emanzipation von einer göttlichen Ordnung ist, muß Prometheus allerdings büßen. Der bestohlene Zeus läßt Prometheus an einen Felsen im Kaukasus schmieden und ein Adler frißt die stets nachwachsende Leber des Angeketteten.

Die gedankliche Figur einer göttlich-natürlichen Ordnung, in die sich der Mensch nicht mehr einfügt, ist alt und mit Anbeginn der Industrialisierung immer wieder in neue Bilder gekleidet worden, die eine „konservative“ Opposition der sich beschleunigenden Moderne entgegen gehalten hat. Sie gehört auch zum ideellen Inventar der Umweltbewegung der 70iger Jahre und hat ihre Wahrnehmung des technischen Fortschritts stark geprägt: er war verknüpft mit Risiken und Umweltschäden und wurde äußerst skeptisch aufgenommen.

Um diese Seite der Umweltbewegung ist es sehr still geworden. Heute überwiegt wieder ein positives Bild des technischen Fortschritts (1). In der öffentlichen Wahrnehmung erscheinen Innovationen nun eher wie ein Zauberstab, mit dem ökonomisch versteinerte Verhältnisse zum tanzen gebracht werden können. Darüber hinaus bergen sie das Versprechen, die hohen politischen Hürden, die einer Weiterentwicklung der Umweltpolitik entgegen stehen, zu unterlaufen. Denn die Verbindung von Umwelt und Innovation gibt Anlaß zu der Vermutung, daß Umweltinnovationen, also Innovationen, die ökologisch vorteilhaft sind (vgl. zur Begriffsklärung den Kasten auf S. 11), eine doppelte oder gar dreifache Dividende abwerfen. Sie verbessern nicht nur die Umweltsituation; sie generieren auch ökonomische Vorteile, zumindest auf Seiten des Unternehmers, der einer solchen Erfindung den Erfolg auf dem Markt verschafft (2). Diese Vorstellung, oft auch als Porter-Hypothese bezeichnet, ist die Hintergrundhypothese einer Reihe aktueller Forschungsprojekte, die sich mit der Verbindung von Innovation und Umwelt befassen. Und sie ist zugleich eine Hoffnung der Umweltpolitik, den derzeitigen Immobilismus zu überwinden. Das macht die Aktualität der thematischen Verknüpfung aus.

\section{Die theoretische Herausforderung}

Sowohl die Umweltproblematik als auch die Innovationsproblematik sind schon seit geraumer Zeit Einfallstore für heterodoxe Theorieansätze in die Bastionen der Neoklassik. Es ist hier nicht der Ort, die Genesis dieser theoriegeschichtlichen Bewegung nachzuzeichnen. Sie hat im Ergebnis dazu gefiuhrt, daß unter den Dächern Ecological Economics und Evolutorische Ökonomik heterodoxe Ansätze organisiert werden, und ferner dazu, daß das ohnehin schon große Gehäuse der neoklassischen Ökonomie mehr verkappte Häretiker beherbergt, als es die mittelalterliche Scholastik jemals vermochte.

Für die Beantwortung der Frage, welche ökonomischen und politische Faktoren das Zustandekommen von Umweltinnovationen begïnstigen oder hemmen, ist das ein gravierendes Problem. Zumindest dann, wenn man sich nicht forschungspragmatisch damit begnïgt, einfache empirische Korrelationen zwischen den Triebkrätten der Innovation und ihren Wirkungen auf die Innovationstätigkeit herauszubekommen, was zweifellos verdienstvoll ist (vgl. die Projektvorstellung von Zundel in diesem Heft). Neoklassisch inspirierte Wirkungsanalysen des Instru- mentensets haben erkennbar Probleme mit der Beschreibung und Erklärung solcher dynamischer Phänomene, die Innovationen nun einmal sind. Umgekehrt läßt die empirische Operationalisierung evolutionstheoretischer Ansäzze auf diesem Feld noch zu wünschen übrig.

Der Versuch einer Integration in Gestalt einer ökonomischen Großtheorie dürfte ein illusionärer und vor allem vermutlich auch ein langweiliger Weg sein. Realistisch ist wohl eher der Weg der wechselseitigen Anregung und Befruchtung, ohne daß die divergierenden methodischen Ansätze verwischt werden. Einige interessante Perspektiven seien kurz angedeutet:

Die umweltökonomische Wirkungsanalyse von Instrumenten ist von gleichsam zeitloser Schönheit. In der Realität der Entwicklung des technischen Fortschritts ist es aber von großer Bedeutung, zu welchem Zeitpunkt ein Instrument oder ein Instrumentenset implementiert wird. Das zeigt schon die Differenzierung in Innovations-, Adaptions- und Diffusionsphase, welche die Heuristik des Forschungsverbundes Innovation und Umweltpolitik prägt (vgl. den Beitrag von Lebr/ Löbbe). Tatsächlich reicht die Bedeutung der Zeit aber noch weiter. Die Unterscheidung zwischen Paradigmenwechseln und inkrementalen Veränderungen deutet ja gleichfalls darauf hin, daß bestimmte technologische Entwicklungslinien zu gewissen Zeitpunkten veränderungsresistent, zu anderen Zeitpunkten aber bereits empfindlich gegenüber kleinen Veränderungen im sektoralen oder branchenbezogenen Innovationssystem sind. Die Identifikation solcher Zeiffenster könnte für die Entwicklung der Umwelt- und Technologiepolitik von großem Interesse sein (vgl. den Beitrag von Erdmann). Eine zweite Forschungsperspektive berührt die Modellierung der makroökonomischen Auswirkungen von Ökosteuern, in der die Produktivitätseffekte, die durch die dynamische Anreizwirkung von Steuern ausgelöst werden, und ihre Folgen auf andere makroökonomische Aggregate wie Beschäftigung und Wachstum i.d.R. nicht angemessen modelliert werden. Hier können „bottom-up"-Studien, die diejenigen Effekte der Technologien erfassen, die durch Ökosteuern in den Bereich der ökonomischen Rentabilität geraten, ebenso interessant sein, wie eine Dynamisierung der makroökonomischen Modelle (3).

\section{- Umweltpolitische Konsequenzen}

Die Begründung für eine Technologie- und Umweltpolitik mit ökologischen Vorzeichen vari- 
iert. Im Prinzip lassen sich drei Argumentationstypen unterscheiden. Da ist erstens das klassische wohlfahrtstheoretische Argument eines doppelten Externalitätenproblems (vgl. dazu Lehr /Löbbe).

Da ist zweitens das Argument einer doppelten Dividende, wonach Umweltinnovationen eben auch eine ökonomische „Dividende“ abwerfen. Hier lassen sich zwei Spielarten unterscheiden. Eine Variante sieht diese Dividende vor allem in Wettbewerbsvorteilen der innovierenden Unternehmen (das ist im Kern die Porter-Hypothese). Die andere Variante sieht daneben oder darïber hinaus makroökonomische Vorteile in Gestalt von Produktivitätszuwächsen und Beschäftigungsimpulsen (Die Umweltbranche ist einer der wenigen Wachstumsbranchen im sektoralen Mix in Deutschland.).

Die Indizienbeweise für oder gegen die PorterHypothese - und mehr ist es beim gegenwärtigen Stand der Forschung noch nicht - sind bislang dünn. Wenn man annimmt, daß die These einer doppelten Dividende zutreffend ist, ist das noch keine hinreichende Bedingung für ein Unterneh- men, eine innovative ökologische Unternehmenspolitik $\mathrm{zu}$ betreiben. Falls sich nämlich herausstellt, daß eine Innovationsstrategie, die auf eine Berücksichtigung von Umweltaspekten verzichtet, eine höhere ökonomische Dividende abwirft, dann hat das Unternehmen, das die Verheiratung von Innovationsmanagement und ökologischer Unternehmenspolitik als dominante Strategie wählt, ein Wettbewerbsproblem. Wirklich triftig wäre die Porter-Hypothese also nur, wenn sich nachweisen ließe, daß unter den wirtschaftlichen Strategien eines Unternehmens die ,ökologische“ zu den besten gehört. Dieser Nachweis - und es sei sofort hinzugefügt, auch der Nachweis des Gegenteils - dürfte in einer Welt der Unsicherheit über die Folgen dieser Strategiewahl extrem schwierig zu führen sein. Weil sich bei der PorterHypothese nicht der Eindruck verfestigen sollte, daß sie zu schön ist, um wahr zu sein, wäre das Zusammentragen von indirekter oder direkter empirischer Evidenz in die eine oder andere Richtung, die über das Zusammentragen von Unternehmensanekdoten hinausreicht, ein wis- senschaftlicher Fortschritt. Dazu gehört natürlich auch die Frage, welche Rolle das Phänomen der Unsicherheit in Verbindung mit den gewählten technischen Trajektorien für die Strategiewahl von Unternehmen spielt.

\section{- Innovationsfreundliche Regulati- onsmuster: Bedeutung von Zielen...}

Faktisch ist die politische Notwendigkeit einer Förderung der Umweltinnovationen in Deutschland und auf der Ebene der EU weitgehend akzeptiert. Sobald jedoch das Ausmaß der ,ökologischen Auslenkung" technologischer Trajektorien in das Blickfeld gerät, ist dieser Konsens briichig. Hübner weist in seinem Beitrag sicher zu Recht darauf hin, daß die Redeweise von Umwelttechnologien als Querschnittstechnologien das Problem verharmlost. Gerade an der Frage, wie im Jahre 2050 die Energieversorgung gesichert werden soll, kann man gut erkennen, daß es da möglicherweise um einen ,radikalen Paradigmenwechsel" des technischen Fortschritts gehen könnte (vgl. Hübner).
D Von Stefon Zundel Ke Klärung des Begiffs der Umweltinnovation ist keineswegs trivial. Erstens gibt es einen Trend, den Innovationsbegriff auszuweiten. Weil technische Innovationen im Unternehmen fast immer begleitet sind von organisato. rischen Veränderungen, kann man diese ebenfalls zu den Innovationen zählen. Von hier ist es nur noch ein kleiner Schritt zur Subsumtion von Neverungen in der Unternehmensstrategie und der Unternehmenskutur unter den Innovationsbegriff. Werden solche soziclen Neverungen als Innovationen akzeptiert, dann ist auch der Gedanke naheliegend, soziale Neverungen einzubeziehen, die ouferhob des Unternehmenssektors angesiedelt sind; vor allem institutionelle Neverungen (formelle und informelle Regeln der sozialen lnteraktion) und dorüber hinous Neuerungen der individuellen Wertvorstellungen und Verhatensweisen. Diese Ausweitung ist ouch und gerode für den Nachholigkeits. diskurs relevant, weil wichtige Schritte in Richrung Nochhaltigkeit auch van den letztgenannten Neverungen eingeleitet werden können (vgl. den Artikel von Lehr und Löbbe in diesem Heft).

Für die Gestaltung von Forschungsprojekten birgt diese durchous plousible Erweiterung des zU erklärenden Gegenstandsbereiches jedoch Probleme. Eine solche Aufblähung des Explanandum ist nur um dem Preis eines verwaschenen Explanans zu haben. Die Erkläung von sozialen Neverungen oder eines Wertewandels ist zweifellos mindestens genouso bedeutsam für das Thema Umwelt und Innovation wie technische Neuerungen; ihre Subsumtion unter den Begriff Innovation fuhrt allerdings zu ziemlich artifiziellen Übertragungen von ökonomischen Erklärungsmustern ouf

\section{Stichwort: Umweltinnovation}

soziale und sozialpsychologische Sachverhalte. Gleichwohl ist die bisherige Debatte um Umwelt und Innovation technikzentriert; die Befassung mit institutionellen Innovationen und dem Wertewandel und ihrer Verbindung zur Umwelt stehen noch ouf der aktuellen forschungspolitischen Agendo.

Eine zweiter Diskussionsstrang ist an die Bestimmung des Atributes „Umweit" im Begriff Umweltinnovation geknüpft. Im Prinzip kann man sich verschiedene Möglichkeiten vorstellen, hier eine definitorische Eingrenzung von Innovationen vorzunehmen. Intentionale Definitionen heben i.d.R. ouf solche Formulierungen ab, wonach es sich um Innovationen handeln müsse, die vom Menschen verursachte Umweltnutzungen oder umweltbezogene Externalitäten vermindern. Do der Nachweis einer solchen Verminderung angesichts der vielfältigen ökologischen Wirkungen, die von einer Innovation ausgehen können, oft recht schwierig ist, begnügen sich die meisten Untersuchungen zum Thema mit einer Aufzählung von Techniken, die gemessen am "common sense" des wissenschaftlichen Fachverstandes als „umwelffreundlich" oder schärfer als sogenannte integrierte Techniken gelten können.

Forschungspragmatisch macht das Sinn, aber dieses Vorgehen kappt die Verbindungen zur Technologiefolgenab- schätzung und zur ökobilanziellen Bewertung von neven Technologien. In aller Regel sind jedoch neve umweltfreundliche Technologien lediglich als Problemlöser für ein singuläres okologisches Problem konzipiert. Ökologische Trade-offs geraten so cus dem Blickfeld der Diskussion, und das birgt die Gefahr, doê die lösung des einen Problems zur Generierung von neven oder Verschärfung von anderen ökologischen Problemen führt. Die Gentechnologie kann beispielsweise erheblich zur Energieeffizienz chemischer Prozesse beitragen, birgt ober dofür andere Risiken. Gerode wenn stoatliche lnstitutionen sich onheischig machen, bestimmie Technologien zu fordern und damit technologische Weichenstellungen vorzunehmen, ist eine solche Folgenabschätzung der ökologischen Trade-offs unverzichibar.

Als weiteres Definitionsmerkmal wird in vielen Untersuchungen - ohne dab dies explizil gemacht wird - ouch die Motivation der Innovateure herangezogen. Demnach wären Umweltinnovationen nur solche Neverungen, die auch in der Absicht auf den Markt gebracht werden, Okologische Verbesserungen herbeizuführen. Damit einher geht eine gravierende Einschränkung der Betrachtungsperspektive. Viele Innovationen sind sicher aus ganz anderen als ökologischen Motiven implementiert worden. Gleichwohl dürften ithre ökologischen Wikungen koum zu unter. schätzen sein (vgl. den Artikel von Erdmann). Folglich macht es durchous Sinn, ouch umgekehrt zu fragen, wie die "normale" Innovationstätigkeit ökologischen Kriterien unterworfen werden kann (vgl. den Arrikel von Rickert und Nill). 
Die effiziente Ausgestaltung einer solchen Politik wie auch die Bedingungen ihres ökologischen Erfolgs sind jedoch Gegenstand einer umfangreichen wissenschaftlichen (und politischen) Diskussion. Die umweltökonomische Instrumentendebatte hat durch die systematische Befassung mit dem Problem der Umweltinnovationen einige interessante neue Impulse bekommen (vgl. dazu die Artikel von Blazejczak et al. und Lehr/Löbbe). Einige Überlegungen seien herausgehoben: Erstens bestätigen Studien aus der hier skizzierten Debatte den Befund, daß die Festlegung von ökologischen Lenkungszielen vor der Wahl der Instrumente eine erfolgreiche Umweltpolitik, d.h. hier die Implementation von Umweltinnovationen, befördern kann. Da die Instrumentendebatte in Deutschland, und nicht nur hier, immer unter taktischen Gesichtspunkten mit Blick auf die eigene Betroffenheit geführt wird, wäre das sicher ein Fortschritt.

\section{...und Kontextbedingungen}

Zweitens beeinflussen die Kontextbedingungen, zu denen neben ökonomischen Bedingungen wie Marktform, Investitionszyklen usw. auch der Politikstil und die Regulationsmuster zählen, in hohem Maß die Wirkung einzelner Instrumente oder Instrumentkombinationen. Im FIU-Projekt ist dafïr mit Blick auf die treibenden Faktoren von Umweltinnovationen die Bezeichnung „MultiImpuls-These“ geprägt worden (vgl. Lehr/Löbbe). Auch wenn damit einmal mehr deutlich geworden sein dürfte, daß es das Königsinstrument nicht gibt, ist damit sicher nicht das letzte Wort in der umweltökonomischen Instrumentendebatte gesprochen. Denn die logische Folge aus diesem Befund ist ja nicht die, daß sich über die Wirksamkeit von Instrumenten oder Instrumentenkombinationen keine wissenschaftlich haltbaren Aussagen mehr machen lassen, sondern nur die, daß die Aussagen zum Kontext des Instrumenteneinsatzes in Beziehung gesetzt werden müssen. Das macht eine einschlägige Politikberatung anspruchsvoller weil komplexer, aber nicht obsolet. Die anwendungsorientierte Forschung wird sich darauf einstellen müssen. Daruiber hinaus erhöht dieses Ergebnis nicht nur die Anforderungen an die wissenschaftliche Politikberatung, sondern auch an die Steuerungskompetenz der staatlichen Akteure. Folglich geraten die politökonomischen und politischen Bedingungen der Implementation von Zielen und Instrumenten wieder stärker in den Fokus der Forschung.
$\mathrm{Zu}$ den Kontextbedingungen zählt auch, daß die Bedingungen für Umweltinnovationen von Branche zu Branche und von Land zu Land stark differieren (vgl. den Beitrag von Hemmelskamp). Eine erfolgreiche ökologische Technologiepolitik wird demnach bei der Festlegung der Ziele und der Mittel diese Bedingungen zu beachten haben. Forschungspolitisch hat dies zur Folge, daß die Untersuchung von nationalen, firmenbezogenen und branchenbezogenen Innovationssystemen interessant wird (vgl. Hemmelskamp und Rickert/Nill). Diese Art von Feinsteuerung einer ökologischen Technologiepolitik erfordert schon wegen der Informationsasymmetrie zwischen Steuerungssubjekt und Steuerungsobjekt eine enge Kooperation zwischen den staatlichen Akteuren einerseits und den Unternehmen und privaten Haushalten andererseits, hat aber ein anrïchiges politisches Odeur, weil die Grenze zwischen dem Allgemeinwohl und den Partikularinteressen nur all$\mathrm{zu}$ leicht verwischt werden kann.

Hier steht das Steuerungsverständnis zur Diskussion. Daß freiwillige Vereinbarungen ohne eine glaubhafte Androhung des Einsatzes harter Instrumente nicht sehr effektiv sein werden, gilt als gesichert. Gleichwohl deckt der Stand der spieltheoretischen Modellierung der freiwilligen Vereinbarungen keineswegs alle sozialen Interaktionen $a b$, die gegenwärtig in der Grauzone zwischen hoheitlichen Akten und privatwirtschaftlichen Handlungen $\mathrm{zu}$ beobachten sind. Eine - politologisch aufgeklärte - Erforschung der Bedingungen der Möglichkeit ökologischer Technologiepolitik ist sicher geboten.

Drittens gilt auch für den Instrumenteneinsatz, daß der kombinierte Einsatz in der Wirkung anders zu beurteilen ist als durch eine schlichte Summierung der Wirkungen jedes einzelnen Instrumentes. Ökonomische Instrumente können sich in ihrer Wirkung wechselseitig verstärken aber ebenso auch wechselseitig blockieren. Insbesondere könnte eine Kombination von ökologischer Technologiepolitik und relativ schwachen Abgaben eine Lenkungswirkung entfalten, die bei einer harten Abgabe, die aber politisch nicht durchsetzbar ist, auch zu erwarten wäre (vgl. dazu den Artikel von Linscheidt). Die Wirkung der sogenannten weichen Instrumente auf das Innovationsverhalten sind in der Diskussion bislang noch recht stiefmütterlich behandelt worden. Die Palette reicht von der Implementation von Managementinstrumenten wie dem EG-Öko-Audit-System (vgl. den Beitrag von Kottmann in diesem Heft) ïber das Stoffstrommanagement bis hin zur Bedeutung von Kommunikation und Organisationsentwicklung.

\section{Schlußbetrachtung}

Rückblickend ist man immer klüger und es ist billige ideologische Konfektionsware, manche technikfeindlichen Vorstellungen aus den 70iger Jahren als vormodern abzuqualifizieren. Die moderne Idee, wonach der technische Fortschritt die Menschheit von Leiden, Krankheit und Tod erlösen könnte, hat sich ja gleichfalls als irrig erwiesen. Niemand wird ernstlich die Segnungen des technischen Fortschritts in Frage stellen. Glïcklicherweise kann er uns aber nicht von den natïrlichen Beschränkungen unserer menschlichen Existenz erlösen. Goethe trifft vielleicht die richtige Mitte, wenn er seinen Prometheus zu Zeus sagen läßt:

„Wähntest du etwa,

Ich sollte das Leben hassen,

In Wüsten fliehen.

Weil nicht alle Blütenträume reiften ?

Hier sitze ich, forme Menschen

nach meinem Bilde

Ein Geschlecht, das mir gleich sei,

$\mathrm{Zu}$ leiden, zu weinen,

$\mathrm{Zu}$ genießen und zu freuen sich

Und dein nicht zu achte. Wie ich“(4)

\section{Anmerkungen}

(1) Vgl. hierzu exemplarisch Gleich, A. von/ S. Leinkauf / S. Zundel (Hrsg.): Surfen auf der Modernisierungswelle? Ziele, Blockaden und Bedingungen ökologischer Innovation. Marburg 1997.

(2) Maßgeblich für diese Position steht Porter, M.E./ C. van der Linde: Green and Competitive: Ending the Stalemate. In: Harvard Business Review, Sept. 1995, S. 120-134. (3) Umstritten sind hier insb. die Wirkungen auf die Produktivität. Während Walz diese als tendenziell positiv einschätzt, vertriitt Hübner in diesem Heft die gegenteilige Auffassung. Vgl. Walz, R. et al.: Mikroökonomische Fundierung der innovativen Wirkung einer C02-/Energieabgabe. In: Klemmer, Paul (Hrsg.): Innovationen und Umwelt. Berlin 1999, S.276.

(4)Zitiert nach Goethe Werkausgabe, Insel Verlag, Band 1 Gedichte Versepen. Baden-Baden 1977, S. 50.

Der Autor
Dr. Stefan Zundel ist Professor für Öffentliche
Finanzwirtschaft, Energie und Umwelt an der Fach-
hochschule Lausitz und löW-Vorstand.
Kontakr: FH Lausitz, Großenhainer Straße 57,
01968 Senftenberg, Tel. 03573/85-733, Fax -709,
E-mail: zundel@ww. Th-lausilz.de


(c) 20I0 Authors; licensee IÖW and oekom verlag. This is an article distributed under the terms of the Creative Commons Attribution Non-Commercial No Derivates License (http://creativecommons.org/licenses/by-nc-nd/3.o/), which permits unrestricted use, distribution, and reproduction in any medium, provided the original work is properly cited. 